\title{
LGMD BASED NEURAL NETWORK FOR AUTOMATIC COLLISION DETECTION
}

\author{
Ana Silva ${ }^{1}$, Jorge Silva ${ }^{1}$ and Cristina Santos ${ }^{2}$ \\ ${ }^{1}$ Industrial Electronic department, University of Minho, Campus of Azurem, Guimaraes, Portugal \\ ${ }^{2}$ Industrial Electronic department, University of Minho, Campus of Azurem, Guimaraes, Portugal \\ \{ana.silva, jbruno\}@dei.uminho.pt, cristina@dei.uminho.pt
}

\begin{abstract}
Keywords: Bio-inspired model, Lobula Giant Movement Detector neuron, artificial neural networks, collision avoidance.

Abstract: Real-time collision detection in dynamic scenarios is a hard task if the algorithms used are based on conventional techniques of computer vision, since these are computationally complex and, consequently, time-consuming. On the other hand, bio-inspired visual sensors are suitable candidates for mobile robot navigation in unknown environments, due to their computational simplicity. The Lobula Giant Movement Detector (LGMD) neuron, located in the locust optic lobe, responds selectively to approaching objects. This neuron has been used to develop bio-inspired neural networks for collision avoidance. In this work, we propose a new LGMD model based on two previous models, in order to improve over them by incorporating other algorithms. To assess the real-time properties of the proposed model, it was applied to a real robot. Results shown that the LGMD neuron model can robustly support collision avoidance in complex visual scenarios.
\end{abstract}

\section{INTRODUCTION}

Many animals extract salient information from complex, dynamic visual scenes to drive behaviours necessary for survival. Insects are particularly challenging for robotic systems: they achieve their performance with a nervous system that has less than a million neurons and weighs only about 0.1 $\mathrm{mg}$. By this reason, some of these insects provide ideal biological models that can be emulated in artificial systems. These models have the potential to reproduce complex behaviours with low computational overhead by using visual information to detect imminent collisions caused either by a rapidly approaching object or self-motion towards an obstacle.

In locusts, the Lobula Giant Movement Detector (LGMD) is a bilaterally paired motion sensitive neuron that integrates inputs from the visual system, responding robustly to images of objects approaching on a collision course (Gray, John R, et al., 2001) (Rind, 1987) (Gabbiani, et al., 1999) (Gray, et al., 2010). This neuron is responsible for triggering escape and collision avoidance behaviours in locusts. The first physiological and anatomical bio-inspired model for the LGMD neuron was developed by Bramwell in (Rind \& Bramwell, 1996). The model continued to evolve (Blanchard, et al., 2000) (Yue \& Rind, 2006) (Stafford, et al., 2007) (Meng, et al., 2010) and it was used in mobile robots and deployed in automobiles for collision detection. These connectionist models have shown that the integration of on and off channels and feed-forward inhibition can account for aspects of the LGMD neuron looming sensitivity and selectivity when stimulated with approaching, translating and receding objects.

However, further work is needed to develop more robust models that can account for complex aspects of visual motion (Guest \& Gray, 2006). In this article, we are interested in understanding the LGMD models previously proposed by (Yue \& Rind, 2006) as well as the achieved properties of the model described at (Meng, et al., 2010). Thereby, we are interested in integrate the two previous LGMD models, (Yue \& Rind, 2006) and (Meng, et al., 2010), in order to take the advantage of noise immunity proposed in (Yue \& Rind, 2006) and direction sensitivity proposed in (Meng, et al., 2010). 
In a previous study, we implemented the models from (Yue \& Rind, 2006) and (Meng, et al., 2010) and submitted them to relevant simulated visual data sets. This step enabled us to understand some of the literature models limitations in relation to obstacle detection and avoidance.

With this knowledge, we propose a new model to cope with the limitations showed by the models implemented ((Yue \& Rind, 2006) and (Meng, et al., 2010)). The proposed model was validated over a set of different visual scenarios. In order to the LGMD network be used as a robust collision detector for real robotic applications, and based on (Yue \& Rind, 2006), it was proposed a mechanism to enhance the features of colliding objects. The model from (Yue \& Rind, 2006) favours grouped excitation, which normally indicates the presence of an obstacle, and ignores isolated excitation, which can be the result of noise present in the captured image, with selective passing coefficients. The model (Yue \& Rind, 2006) has the capability to filter out the isolated excitations through the excitation gathering mechanism, allowing that only parts of the captured image with bigger excitatory spatial areas can contribute to the excitation of the LGMD cell. Besides this extraordinary capability of noise reduction, when computationally implemented, the neural network based on (Yue \& Rind, 2006) generated false collision alarms when stimulated with receding objects. Based on (Meng, et al., 2010), we have modified the LGMD model, so that it could distinguish approaching from receding movements.

On the other side, the LGMD model proposed by (Meng, et al., 2010) is not immune to the presence of noise levels in the captured image, which can leads it to produce false collision alerts in the presence of noise. However, as it was said before, this model is able to detect the direction of movement in depth. Taking the advantages of each model (Yue \& Rind, 2006) (Meng, et al., 2010), we decided to propose a new LGMD model that is more robust in collision detection. The model here proposed was tested on simulated and non-simulated environments and, through the obtained results, it can be concluded that it works very efficiently in both scenarios. In relation to the real performance of the proposed method, a collision avoidance is judge by the evaluation of a real robot moving around in a real environment and avoiding real obstacles (of different shapes, sizes and colours) and processing captured images (containing real noise, blur, reflections, etc). In our perspective, the new proposed method increases the precision of obstacle detection, in a way that this model is robust to the presence/absence of high noise levels in the captured image, as well as is able to detect the movement direction of the visual stimulus. Besides that, when tested in a real environment, the results were very satisfactory. For a better understanding of the work developed, the paper was organized in the following way: in section 2 , we make a detailed description of the proposed LGMD neural network model. In section 3 are presented some experimental results on simulated and recorded video data. In this section we also present the experiments carried out with a robot DRK8000 to test the stability of this model in relation to collision detection in real scenarios. Finally, in section 4 we make the conclusions of the work here described.

\section{THE PROPOSED NEURAL NETWORK MODEL FOR LGMD}

The biological inspired neural network here proposed is based on previous models described on (Blanchard, et al., 2000) (Yue \& Rind, 2006) (Stafford, et al., 2007) (Meng, et al., 2010). The modified neural network is shown on Figure 1.

The LGMD neural network here proposed is composed by five groups of cells: photoreceptor cells (P layer), excitatory cells (E layer), inhibitory cells (I layer), summing cells (S layer) and noise reduction cells (NR layer). Besides that, it is composed by five single cells: the direction sensitive system, composed by the approaching cell (A cell), the receding cell ( $\mathrm{R}$ cell) and the direction cell (D cell), the feed-forward inhibition cell (FFI cell) and the LGMD cell.

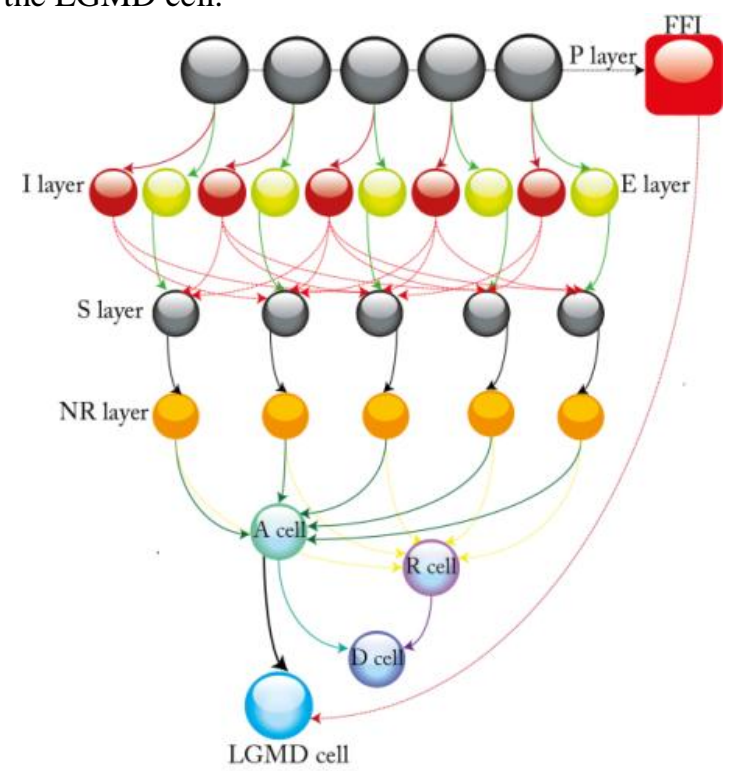

Figure 1: Schematic illustration of the proposed LGMD model. There are five groups of cells and five single cells: P layer: photoreceptor cells; E layer: excitatory cells; I 
layer: inhibitory cells; S layer: summing cells; NR layer: noise-reduction cells; A cell: approaching cell; $\mathrm{R}$ cell: receding cell; D cell: direction cell; FFI cell: feed-forward inhibition cell. LGMD cell: represents the LGMD biological neuron.

A grayscale image of the camera current field of view, represented has a matrix of values (from 0 to $255)$, is the input to a matrix of photoreceptor units (P layer). This layer calculates the absolute difference between the luminance of the current and the previous input image, mathematical represented by the following equation:

$$
P_{f}(x, y)=\left|L_{f}(x, y)-L_{f-1}(x, y)\right|
$$

Where $P_{f}$ is the output of the P layer at frame $\mathrm{f}$, $L_{f}$ and $L_{f-1}$ are the captured luminance at frames $f$ and $\mathrm{f}-1$, respectively. The output of the $\mathrm{P}$ layer is the input of two layers: the excitatory (E) and the inhibitory (I) layers. To the excitatory cells of the E layer, the excitation that comes from the $\mathrm{P}$ layer is passed directly to the retinotopic counterpart. The inhibition layer (or I layer) receives the output of the $P$ layer and applies a blur effect on it, using:

$$
\begin{gathered}
I_{f}(x, y)=1 / 9 \sum_{i=-1}^{1} \sum_{j=-1}^{1} \delta(x+\mathrm{i}, \mathrm{y}+\mathrm{j}) \\
\delta(\mathrm{x}, \mathrm{y})=P_{f-1}(x, y)
\end{gathered}
$$

Where $I_{f}$ is the output of the I layer at frame f, and $P_{f-1}$ is the output of the P layer at frames $\mathrm{f}-1$. Then, the output of the I layer passes to the summing layer, in a retinotopic mode.

The summing layer (or $S$ layer) receives the output from the $\mathrm{E}$ and I layers and performs the following operation:

$$
\begin{gathered}
S_{f}(x, y)=E_{f}(x, y)-\mathrm{I}_{\mathrm{str}} \cdot I_{f}(x, y) \\
E_{f}(x, y)=P_{f}(x, y) \text { and } S_{f}(x, y) \geq 0
\end{gathered}
$$

Where $P_{f}$ is the output of the P layer at frame $\mathrm{f}, I_{f}$ the output of the I layer at frame $f$ and $I_{\text {str }}$ (a scalar, set to be 0.35) represents the inhibition strength.

Based on (Yue \& Rind, 2006), it was added a new mechanism for the LGMD neural network to filter the background noise. This mechanism, implemented in the NR layer, takes clusters of excitation in the $S$ units to calculate the input to the LGMD membrane potential. These clusters provide higher individual inputs then the ones of isolated $\mathrm{S}$ units. The excitation that comes from the $S$ layer is then multiplied by a passing coefficient $C e_{f}$, which value depends on the surrounding neighbours of each pixel, calculated as follows:

$$
\begin{gathered}
C e_{f}(x, y)=\frac{1}{9} \sum_{i=-1}^{1} \sum_{j=-1}^{1} \delta(\mathrm{x}+\mathrm{i}, \mathrm{y}+\mathrm{j}) \\
\delta(\mathrm{x}, \mathrm{y})=S_{f-1}(x, y)
\end{gathered}
$$

The final excitation level of each cell in the NR layer, at frame $\mathrm{f}\left(N R_{f}\right)$, is given by:

$$
\begin{gathered}
N R_{f}(x, y)=\left|S_{f}(x, y) \cdot C e_{f}(x, y) \cdot \mathrm{w}^{-1}\right| \\
\mathrm{w}=\Delta \mathrm{c}+\max \left(\left|[C e]_{f}\right|\right) \cdot \mathrm{C}_{\mathrm{w}}^{-1}
\end{gathered}
$$

$C w$ is set to 4 , and $\Delta \mathrm{c}$ is a small number $(0.01)$, to prevent $w$ from being zero, and $\max \left(\mid[C e]_{f}\right)$ is the largest element in matrix $\left|[\mathrm{Ce}]_{f}\right|$.

Within the NR layer, a threshold filters the decayed excitations (isolated excitations), as:

$$
\widetilde{N R}_{f}(x, y)=\left\{\begin{array}{c}
N R_{f}(x, y), \text { if } N R_{f}(x, y) \cdot C_{d e} \geq T_{d e} \\
0, \text { if } N R_{f}(x, y) \cdot C_{d e}<T_{d e}
\end{array}\right.
$$

Where $C_{d e} \in[0,1]$ is the decay coefficient and $T_{d e}$ is the decay threshold (set to 20). The decay threshold here used was experimentally determined. The NR layer is able to filter out the background detail that may cause excitation. Hence, only the main object in the captured scene will cause excitation.

The LGMD potential membrane $K_{f}$, at frame f, is summed after the NR layer, as described in the following equation:

$$
L G M D_{f}=K_{f}=\sum_{x=1}^{n} \sum_{y=1}^{m}\left(\widetilde{N R}_{f}(x, y)\right)
$$

Where $n$ is the number of rows and $m$ is the number of columns of the matrix representing the captured image.

The A and R cells (adapted from (Meng, et al., 2010)) are two grouping cells for depth movement direction recognition. The A cell holds the mean of three samples of the LGMD cell:

$$
A_{f}=\left(K_{f-1}+K_{f-2}+K_{f-3}\right) / 3
$$

The $R$ cell shares the same structure as the A cell but with a temporal difference, having one frame delay from $\mathrm{A}$.

$$
R_{f}=A_{f-1}
$$


Analyzing the equations above described, it can be concluded that if the object is approaching $A_{f}>R_{f}$ and if the object is receding, $R_{f}>A_{f}$.

The $\mathrm{D}$ cell is used to calculate the direction of movement. This can be represented by the following equation:

$$
D_{f}=a b s\left(A_{f}\right)-a b s\left(R_{f}\right)
$$

This cell exploits the movement direction in depth. It is based on the fact that a looming object (approaching) gets larger whereas a receding object gets smaller. In a way to distinguish the movement direction detected by the $\mathrm{D}$ cell, it was added a threshold mechanism, $T_{D}$ (set to $0.05 \times n \times m$ ), where $n$ is the number of rows and $m$ is the number of columns of the captured image), which was experimentally determined.

$$
D_{f}=\left\{\begin{array}{c}
1, \text { if } D_{f} \geq T_{D} \\
0, \text { if } T_{D}>D_{f}>-T_{D} \\
-1, \text { if } D_{f} \leq-T_{D}
\end{array}\right.
$$

The LGMD membrane potential $K_{f}$ is then transformed to a spiking output using a sigmoid transformation,

$$
k_{f}=\left(1+e^{-K_{f} n_{c e l l}^{-1}}\right)^{-1}
$$

Where $n_{\text {cell }}$ is the total number of cells in the NR layer and $k_{f} \in[0.5,1]$. The collision alarm is decided by the spiking of the LGMD cell.

However, the spiking output $k_{f}$ (from equation (15), representative of the LGMD cell output) is not the final output of the neural network. It was implemented a spiking mechanism using an adaptable threshold. This threshold starts with a value experimentally determined, $T_{s}(0.88)$ and it is updated at each frame, through the following process,

$$
T_{s}=\left\{\begin{array}{c}
T_{s}+\Delta t, \text { if } s_{a v}>\mathbb{I} \text { and }\left(T_{s}+\Delta t\right) \in\left[T_{l}, T_{u}\right] \\
T_{s}-\Delta t, \text { if } s_{a v}<\mathbb{I I} \text { and }\left(T_{s}-\Delta t\right) \in\left[T_{l}, T_{u}\right] \\
T_{s}, \text { otherwise }
\end{array}\right.
$$

Where $\left[T_{l}, T_{u}\right.$ ] defines the lower and upper limits for adaptation ( $T_{l}$ is 0.80 and $T_{u}$ is 0.90$), \Delta t$ is the increasing step (0.01), III $(0.72)$ is a threshold that limits the averaged spiking output $s_{a v}$, between frame $f$ - $n$ to frame $f-k$ ( $n$ is 5 and $k$ is 2 ),

$$
s_{a v}=\frac{1}{n-k+1} \sum_{i=k}^{n} S_{f-i}
$$

If the sigmoid membrane potential $k_{f}$ exceeds the threshold $T_{s}$ a spike is produced, as follows:

$$
S_{f}=\left\{\begin{array}{c}
1, \text { if } k_{f} \geq T_{s} \\
0, \text { other }
\end{array}\right.
$$

Finally, a collision is detected when there are $n_{s p}$ spikes in $n_{t s}$ time steps $\left(n_{s p} \leq n_{t s}\right)$, where $n_{s p}$ is 4 for the simulated experiments and 3 to real experiments (since captured images in real experiments present higher variations) and $n_{t s}$ is 5 for all the experiments (values experimentally determined).

$$
C_{f}=\left\{\begin{array}{c}
1, \text { if } \sum_{\substack{f-n_{t s} \\
0, \text { other }}}^{f} S_{f} \geq n_{s p} \\
\text {. }
\end{array}\right.
$$

The robot escape behavior is initialized when a collision is detected. Additionally, the spikes can be suppressed by the FFI cell when occurs an intense field movement. When the robot is turning, sudden changes in the visual scenario occur which can lead the network to produce spikes and even false collision alerts.

The feed-forward inhibition cell (FFI cell) is very similar to the LGMD cell but the FFI cell receives the output from the $\mathrm{P}$ layer (and not from the NR layer), being represented by:

$$
F F I_{f}=\frac{\sum_{x=1}^{n} \sum_{y=1}^{m}\left|P_{f-1}(x, y)\right|}{n c e l l}
$$

Where $F F I_{f}$ is the output of the FFI cell at frame $\mathrm{f}$ and $P_{f-1}$ is the output of the P layer at frame $\mathrm{f}-1$. If $F F I_{f}$ exceeds a threshold $\mathrm{T}_{\mathrm{FFI}}$, the spikes produced by the LGMD cell are inhibited. The threshold $\mathrm{T}_{\mathrm{FFI}}$ was experimentally determined (set to 25).

As described in this section, the proposed neural network for the LGMD neuron only involves low level image processing. So, the proposed neural network model is able to work in real time and, besides that, is independent of object classification. 


\section{EXPERIMENTAL RESULTS ON THE PROPOSED MODEL}

In a way to test the efficiency of the LGMD neural network here proposed, two different data sets were used. The first experiment was made on a simulated data set and, after that, it was used a recorded video to prove the capacity of the LGMD neural network here proposed to work in a real environment. In the second experiment, we implemented the LGMD neural network in a real robot, DRK8000, located within a real arena. All the parameters were kept the same during all the experiments.

\subsection{Simulated Environment}

We develop a simulation environment in Matlab (MATLAB, 2011) that enables us to assess the effectiveness of the proposed LGMD neural network. Objects were simulated according to their movement and the corresponding data was acquired by a simulated camera and processed by the LGMD neural network. Image sequences were generated by a simulated camera with a field of view of $60^{\circ}$ in both $x$ and $y$ axis and a sampling frequency of 100 Hz. The simulated environment enabled us to adjust several parameters, such as: image matrix dimensions, the camera rate of acquisition, the image noise level, the object shape, the object texture, as well as other parameters.

The computer used in the experiments here described was a Laptop (Toshiba Portegé R83010R) with $4 \mathrm{GHz}$ CPUs and Windows 7 operating system. Relative to the parameters used by the LGMD neural network, they were determined before the experiments.

\subsection{Results on simulated data set and on real recorded data}

Previous to the stimulation of the LGMD model here proposed, several experiments have been made in order to verify and analyse how the image of a black squared object grows when it is approaching to a simulated camera. For that, we used synthesized black (0) and white (255) images, with 100 (horizontal) by 100 (vertical) pixels of resolution. The object being observed was a square black filled rectangle (figure 2), whose properties as acquisition frequency, velocity, trajectory, shape, texture, noise level or object size could be changed.

The obtained results enabled us to conclude that the image growing depends on several factors, including the camera acquisition frequency and the object velocity, among other characteristics However, the curve that approximates this growing is always an exponential curve, whose slope depends on these aforementioned factors.

As a second step, and in the context of this study, we made an exhaustive analysis of the response of our LGMD model to a set of standard LGMD stimulation protocols, which allowed us to validate our model with respect to the biological system (Gabbiani, et al., 2001). In our first experiment we evaluated the proposed LGMD model, by using a looming stimulus consisting of a solid square with 10 repetitions to each size/velocity $=l /|v|$ pair (where $l$ stands for the half length of the square object and $v$ for its linear velocity). With these experiments, we wanted to prove that our model respects the properties verified by Gabbiani et al. (Gabbiani, et al., 1999) (Gabbiani, et al., 2001) as well as by Badia (Badia, et al., 2010). These properties, founded in the locust visual system, include a linear relation between the time of the peak firing rate of the LGMD neuron and the ratio that correlated the stimulus object size $(l)$ and the stimulus linear velocity ( $v$ ) (Gabbiani, et al., 2002).

As a first step, we analysed the LGMD model here proposed using, for that, a looming stimulus in the form of a black square. We repeated this procedure to ten different $l \backslash v \mid$ ratios, from 5 to 95 milliseconds. Through the obtained results it was observed that the fit of the TTC (time-to-collision) of the peak firing rate, obtained through the LGMD neural network, versus the $l|v|$ ratios, is consistent with the biological results, showing a correlation coefficient $(r)$ superior to 0.99 .

In literature, it was also reported that the LGMD neuron responses are largely independent of the stimulus texture, shape and approaching angle (Badia, et al., 2010) (Gabbiani, et al., 2001). The results obtained when we subjected our model to stimulus with different textures, different shapes and different approaching angles (as shown on Figure 2) to different $l \Lambda v \mid$ ratios (also from 5 to $95 \mathrm{~ms}$ ) showed us that the proposed model still has a linear relationship between the TTC of the peak firing rate versus the $l|v|$ ratios, not being affected by the change on the stimulus characteristics, as reported for the biological system.

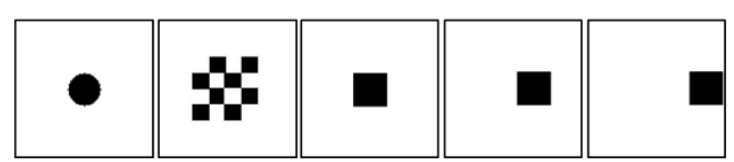

Figure 2: Artificial visual stimuli, developed in Matlab (MATLAB, 2011). 
In the first experiment made, to test the LGMD model invariance to textured objects, the correlation coefficient between the LGMD model responses and the regression line was bigger than 0.99 ( $r>0.99)$, meaning that the LGMD model is not sensitive to the texture of the objects. Relatively to the second experiment, which allowed us to test the LGMD model invariance to object shape, the correlation coefficient between the model responses and the regression line was 0.97 , approximately $(r=0.9734)$. And, for the last one, in order to test the LGMD model invariance to different approaching angles of the looming stimulus, we aligned the camera at different angular orientations relatively to the projection screen. After the analysis of the obtained results we could conclude that, as the camera angle deviates from the center $\left(0^{\circ}\right)$, the correlation coefficient decays (for a camera angle of $33^{\circ}$ of deviation, $r=0.995$; for a camera angle of $55^{\circ}$ of deviation, $r=0.9192$ and, finally, for a camera angle of $75^{\circ}$ of deviation, $r=0.874$ ). Through this validation, we could conclude that the LGMD model here proposed respects the biological principles.

After the model validation, we fed the LGMD neural networks proposed by (Yue \& Rind, 2006) (Meng, et al., 2010) and the one proposed by us, with simulated image sequences (a representation can be seen on Figure 3).

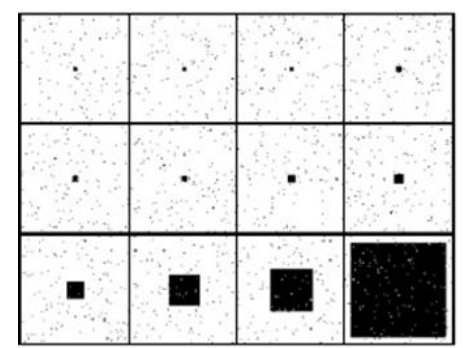

Figure 3: Selected frames from the simulated image sequence. The square object changes its size from small (10 by $10 \mathrm{~cm}, l=5 \mathrm{~cm}$ ) to big, and moves at $100 \mathrm{~cm} / \mathrm{s}$ $(v=100 \mathrm{~cm} / \mathrm{s})$. The relation $l \Lambda v \mid$ is 50 milliseconds. The noise level in all the image sequence is, approximately, 500 pixels. The frame rate was $100 \mathrm{~Hz}$.

In this point, we used four different simulated visual stimuli:

Stimulus 1: composed by a black approaching square, over a white background, with $l /|v|$ equal to 50 milliseconds, acquired with a frame rate of 100 $\mathrm{Hz}$, without noise added to the image sequence.

Stimulus 2: composed by a black receding square, over a white background, with $l|v|$ equal to
50 milliseconds, acquired with a frame rate of 100 $\mathrm{Hz}$, without noise added to the image sequence.

Stimulus 3: composed by a black approaching square, over a white background, with $l|v|$ equal to 50 milliseconds, acquired with a frame rate of 100 $\mathrm{Hz}$, with 500 pixels of noise added to the image sequence.

Stimulus 4: composed by a black receding square, over a white background, with $l|v|$ equal to 50 milliseconds, acquired with a frame rate of 100 $\mathrm{Hz}$, with 500 pixels of noise added to the image sequence.

In addition to these four simulated visual stimuli, and in order to test the LGMD models in a real environment, we recorded a real video sequence, using a Sony Cyber shot digital camera 7.2 megapixels to obtain the video clip. The resolution of the video images was 640 by 480 pixels, with an acquisition frequency of 30 frames per second. In Figure 4 it is represented some selected frames captured by the camera, showing a real approaching black ball.

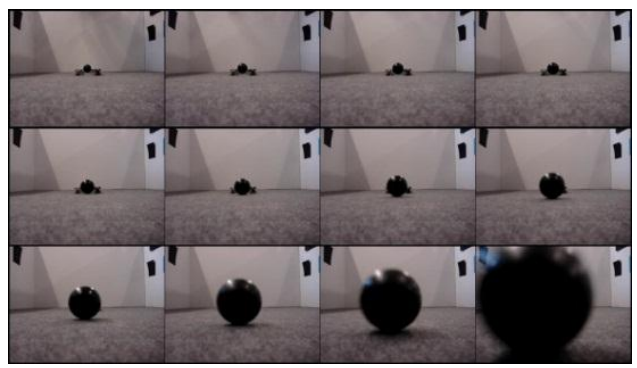

Figure 4: Selected frames from the recorded image sequence used in the experiment. The recorded video is composed by 44 frames, showing a black approaching ball.

After the computational implementation of the LGMD models proposed in (Yue \& Rind, 2006) and (Meng, et al., 2010), and after subject those to all the stimuli previously described, we verify that the collisions were detected, by the different LGMD models, at different time instants and, consequently, at different distances of the object (simulated or real) relatively to the camera. For a better understanding and organization of the results, we decided to call "LGMD model 1" to the model proposed by (Yue \& Rind, 2006) and "LGMD model 2" to the model proposed by (Meng, et al., 2010).

The results obtained are resumed in the following table. 
Table 1: Distances at which collision detection alarms were generated by the LGMD model 1 and LGMD model 2 , in five different situations tested.

\begin{tabular}{|c|c|c|}
\cline { 3 - 3 } \multicolumn{1}{c|}{} & LGMD model 1 & LGMD model 2 \\
\hline Stimulus 1 & $26 \mathrm{~cm}$ & $14 \mathrm{~cm}$ \\
\hline Stimulus 2 & $35 \mathrm{~cm}$ & -- \\
\hline Stimulus 3 & $26 \mathrm{~cm}$ & $20 \mathrm{~cm}$ \\
\cline { 1 - 1 } Stimulus 4 & $35 \mathrm{~cm}$ & $11 \mathrm{~cm}$ \\
\cline { 1 - 1 } Real video & $24 \mathrm{~cm}$ & $14 \mathrm{~cm}$ \\
\hline
\end{tabular}

As we can observe on Table 1, in the approaching situations (stimulus 1, 3 and real video), the LGMD model 1 detected a collision when the object was located at, approximately, 24-26 cm relatively to the camera. This model showed its immunity to the noise presence since it detected a collision exactly at the same distance when stimulated with stimulus 1 (absence of noise) and 3 (presence of high noise levels). However, if we observe the obtained results for the LGMD model 1 when stimulated with receding objects (stimulus 2 and 4) it detected a false collision when the object was located at $35 \mathrm{~cm}$ relatively to the camera, in both situations tested. Through these last results one can conclude that the LGMD model 1 is not able to distinguish between approaching and receding objects, generating false collision alerts in the presence of receding objects. But we can also conclude that this model has high immunity to the noise presence in the captured images.

Relatively to the LGMD model 2 and observing Table 1, for the stimulus 1 and 3, this model did not detect collisions for the same distance. When stimulated with stimulus 1 , it detected a collision when the object was located at $14 \mathrm{~cm}$ relatively to the camera and when stimulated with stimulus 3 , a collision was detected sooner, when the object was at $20 \mathrm{~cm}$ relatively to the camera. This happened due to the fact that the LGMD model 2 is not immune to the noise presence and the noise pixels, which were not eliminated by this model, composed an extra excitation to the LGMD neural network.

In the presence of a receding object, the LGMD model 2 was able to not produce false collision alerts when stimulated with stimulus 2 . However, when we feed the LGMD model 2 with the stimulus 4, it detected a false collision when the object was located at $11 \mathrm{~cm}$ relatively to the camera. This happened also due to the non-immunity of the LGMD model 2 to the noise presence, which works as an extra excitation, leading to the generation of false collision alerts.
After this analysis, relative to the behaviour of the LGMD model 1 and LGMD model 2 in different situations, we could extract some particular characteristics of both models. These results leaded us to produce a mixed LGMD model, combining the advantages of the LGMD model 1 and LGMD model 2. Thus, the LGMD model here proposed provides noise immunity, as well as a directionally sensitive system.

Figure 5 shows the output from the LGMD model here proposed. In this figure, at each time step we can observe the result of different mathematical processing (described on section 2), corresponding to the layers of the proposed model, executed sequentially, necessary to detect, with the maximum precision, an imminent collision.

The analysis of these results showed, on Figure 5 , that the LGMD neural network detected a collision at time -0.19 seconds, i.e., when the object was located at $19 \mathrm{~cm}$ relatively to the camera.

In relation to the receding object, represented on Figure 6, as expected no collisions were detected.



Figure 5: LGMD model response to an approaching object which 1/|v| set at 50 milliseconds. Spike Rate: blue graph: is obtained by the ratio of the $A$ cell value and the total number of cells in the NR layer. Green graph: is obtained by the ratio of the $R$ cell value and the total number of cells in the NR layer. $D$ cell: output of the direction cell: 1: approaching, 0: no significant movement, -1 : receding. Ts: adaptative threshold represented by the red line; the gray points represent the $s_{a v}$ output. $L G M D$ : Blue graph: output of the $L G M D$ cell (mathematically represented by the $k_{f}$ value). Green points: output of the LGMD cell after the Feed-forward inhibition. LGMD after $T_{s}$ : represents the output of the LGMD cell after the application of the threshold $T_{s}$ and being in account the output of the $D$ cell. Collision detected: the output of this graph is one when it is detected four successive spikes in five successive time-steps. In all these graphs, the zero value corresponds to the time of collision. 


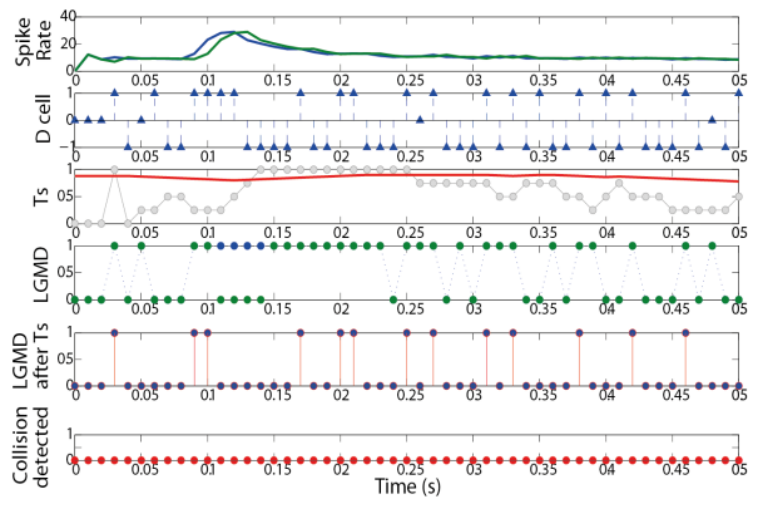

Figure 6: LGMD model response to a receding object which $1 /|\mathrm{v}|$ was equal to 50 milliseconds. The legend of this figure is similar to the one described on the figure 5 .

The results previously described showed the efficacy of the LGMD neural network proposed by us. On Figure 5 and Figure 6, it is shown the LGMD model immunity to high noise levels, as well as the capability of this model in distinguish the direction of movement between successive frames. Then, to test the capability of the proposed LGMD model in a more realistic environment, we subjected it to the real video sequence, represented on Figure 4. In this situation, the model produced a collision alert when the object was located at $28 \mathrm{~cm}$ relatively to the camera.

\subsection{Results on a real robot}

In order to assess the capability of the LGMD model here proposed in a real environment, we used a DRK8000 mobile robot, with a 8-bit CIF (352 by 288 pixels) colour CMOS camera, working at $10 \mathrm{~Hz}$, having a field-of-view of 70 degrees, approximately. The robot was located within an arena, surrounded by four walls with attached objects with different colours, shapes, textures and sizes. The arena has 16 $\mathrm{m}^{2}$. We used the dead reckoning process in order to predict the position of the robot at each time instant.

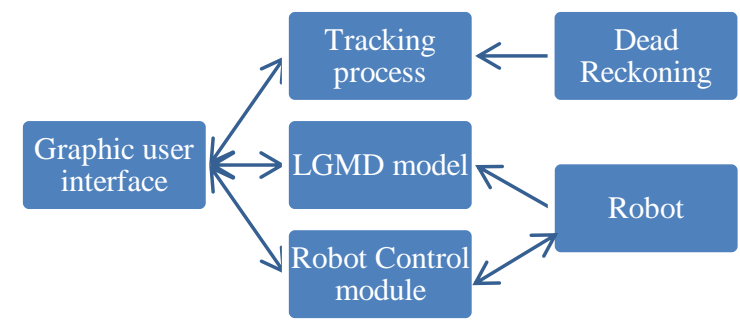

Figure 7: Integrated simulation processes used in the real experiment.
As Figure 7 shows, the simulation system used comprises four processes: The LGMD model module, the robot control module, the tracking module and the graphical user interface. The experiment ran in real-world time, with 10 time steps per second. The LGMD model module was composed by the different layers observed on Figure 1 , and the final output of this model comprises two different states: "collision detected" or "noncollision detected".

The second module, the robot control module, consists in the reactive control structure, capable of controlling the robot, using only the output of the LGMD model module. The behaviours comprised by this module, can be divided in two: 1- basic exploratory activity; 2- collision avoidance of obstacles, triggered by the response of the LGMD module. If the robot detects an imminent collision, it stops, rotates and, then, continues the movement in a straight line. The turning speed is $1 / 3$ of the robot speed for the left wheel and $-1 / 3$ of the robot speed to the right wheel. The robot was set to rotate during 1 second. Finally, in relation to the tracking process, we used dead reckoning in order to determine the position of the robot at each time step and, then, use this information to infer about the distance at which the robot deviates of a potential collision/obstacle.

In the experiment, three long robot movement periods (120 seconds, speed at 5,10 and $15 \mathrm{~cm} / \mathrm{s})$ were conducted to test and show the mechanism of the collision detector in a real environment.

After the experiment, and through the analysis of the dead reckoning relative to the robot movement during all the running time, we could extract, as well as characterize, the collision detections. Collision detections between $20 \mathrm{~cm}$ and $100 \mathrm{~cm}$ away from the wall were classified as correct, those detected closer than $20 \mathrm{~cm}$ from the wall were classified as missed, and collisions detected at a distance over $100 \mathrm{~cm}$ as false positives (see Figure 8). 

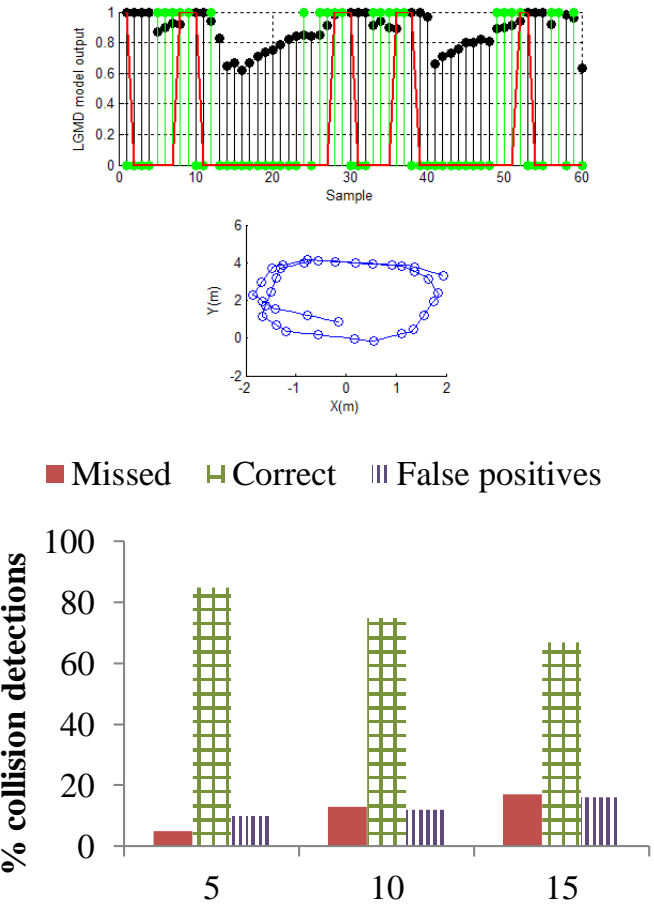

Robot speed $(\mathbf{c m} / \mathbf{s})$

Figure 8: Top graph: LGMD model output, running at real time, for different LGMD layers, during the experiment with the DRK8000 robot, for a robot speed of $5 \mathrm{~cm} / \mathrm{s}$. Midle graph: Dead reckoning of the robot during the initial time steps of the experiment, for a robot speed of 5 $\mathrm{cm} / \mathrm{s}$. Categorization of the collision detections as missed, correct and false positives, for three different robot velocities tested: 5,10 and $15 \mathrm{~cm} / \mathrm{s}$.

As represented on Figure 8, as the velocity of the robot increases, the percentage of collision detections classified as correct decreases, as well as the percentage of missed and false positives detections increases. The increase of missed collisions to higher speeds was due to the simple collision avoidance mechanism adopted in this article: the robot always turns to the same side regardless the relative position of the nearby objects. The increase in the number of false positives to higher velocities is based on the fact that, at higher velocities, the difference between successive frames is higher, leading to the production of high excitation levels and, consequently, a bigger number of collision detection alarms.

Although the difference verified in relation to correct collision detections between different velocities, the results obtained are very satisfactory, as the number of correct detections are always higher than the sum of missed and false positive detections.

\section{CONCLUSIONS}

In this paper, we propose a modified LGMD model based on the identified LGMD neuron of the locust brain. The model proved to be a robust collision detector for autonomous robots. This model has a mechanism that favours grouped excitation, as well as two cells with a particular behaviour that provide additional information on the depth direction of movement.

For applications as collision detectors in robotics, the model proposed is able to remove the noise captured by the camera, as well as enhance its ability to recognize the direction of the object movement and, by this way, remove the false collision alarms produced by the previous models when a nearby object is moving away.

Experiments with a DRK8000 robot showed that with these two new procedures, the robot was able to travel autonomously in real time and within a real arena.

The results illustrate the benefits of the LGMD based neural network here proposed, and, in the future, we will continue to use and enhance this approach, using, for that, a combination of physiological and anatomical studies of the locust visual system, in order to improve our understanding about the relation between the LGMD neuron output and the locust muscles related to the avoidance manoeuvres.

\section{ACKNOWLEDGEMENTS}

This work is funded by FEDER Funds through the Operational Programme Competitiveness Factors COMPETE and National Funds through FCT Foundation for Science and Technology under the Project: FCOMP-01-FEDER-0124-022674.

Ana Silva is supported by $\mathrm{PhD}$ Grant SFRH/BD/70396/2010, granted by the Portuguese Science Foundation.

\section{REFERENCES}

Badia, S. B. i., Bernardet, U. \& Verschure, P. F. M. J., 2010. Non-Linear Neuronal Responses as an Emergent Property of Afferent Networks: A Case Study of the 
Locust Lobula Giant Movement Detector. PLoS Comput Biol, 6(3), p. e1000701.

Blanchard, M., Rind, F. C. \& Verschure, P. F. M. J., 2000. Collision avoidance using a model of the locust LGMD neuron. Robotics and Autonomous Systems, 30(1), pp. 17-37.

Gabbiani, F., Krapp, H. G., Koch, C. \& Laurent, G., 2002. Multiplicative computation in a visual neuron sensitive to looming. Nature, Volume 420, pp. 320324.

Gabbiani, F., Krapp, H. \& Laurent, G., 1999. Computation of object approach by a wide-field motion-sensitive neuron. J. Neurosci., Volume 19, 1122-1141.

Gabbiani, F., Mo , C. \& Laurent, G., 2001. Invariance of Angular Threshold Computation in a Wide-Field Looming-Sensitive Neuron. The Journal of Neuroscience, 21(1), p. 314-329.

Gray, John R , J. R., Lee, J. K. \& Robertson, R., 2001. Activity of descending contralateral movement detector neurons and collision avoidance behaviour in response to head-on visual stimuli in locusts. Journal of Comparative Physiology A, Volume 187, pp. 115129.

Gray, J. R., Blincow, E. \& Robertson, R., 2010. A pair of motion-sensitive neurons in the locust encode approaches of a looming object. Journal of Comparative Physiology A: Neuroethology, Sensory, Neural, and Behavioral Physiology, 196(12), pp. 927938.

Guest, B. B. \& Gray, J. R., 2006. Responses of a loomingsensitive neuron to compound and paired object approaches. Journal of Neurophysiology, 95(3), pp. 1428-1441.

MATLAB, 2011. version 7.12.0 (R2011a). Natick, Massachusetts: The MathWorks Inc..

Meng, H., Yue, S., Hunter, A. Appiah, K., Hobden, M. Priestley, N., Hobden, P. \& Pettit, C., 2009. A modified neural network model for the Lobula Giant Movement Detector with additional depth movement feature. Atlanta, Georgia: Proceedings of International Joint Conference on Neural Networks, 14-19 June.

Rind, F., 1987. Non-directional, movement sensitive neurones of the locust optic lobe. Journal of Comparative Physiology A: Neuroethology, Sensory, Neural, and Behavioral Physiology, Volume 161, pp. 477-494.

Rind, F. C. \& Bramwell, D. I., 1996. Neural Network Based on the Input Organization of an Identified
Neuron Signaling Impeding Collision. Journal of Neurophysiology, 75(3), pp. 967-985.

Stafford, R., Santer, R. D. \& Rind, F. C., 2007. A bioinspired visual collision detection mechanism for cars: combining insect inspired neurons to create a robust system. BioSystems, Volume 87, pp. 164-171.

Yue, S. \& Rind, F.C., 2006. Collision detection in complex dynamic scenes using an LGMD-based visual neural network with feature enhancement. IEEE transactions on neural networks. 17 (3), 705-716. ISSN 1045-9227. 